\title{
SUFFICIENT CONDITIONS FOR NONOSCILLATION OF A SECOND ORDER NONLINEAR DIFFERENTIAL EQUATION
}

\author{
JOHN R. GRAEF ${ }^{1}$ AND PAUL W. SPIKES ${ }^{1}$
}

ABSTRACT. Using a generalization of a variational theorem due to Leighton, the authors obtain sufficient conditions for the second order nonlinear differential equation $\left(a(t) x^{\prime}\right)^{\prime}+q(t) f(x)=r(t)$ to be nonoscillatory. Examples showing the necessity of certain hypotheses are also given.

1. Introduction. In [1] Komkov generalized a well-known variational theorem of Leighton [2]. In this paper we apply Komkov's result to obtain sufficient conditions for all solutions of the second order nonlinear equation $\left(a(t) x^{\prime}\right)^{\prime}+q(t) f(x)=r(t)$ to be nonoscillatory. Few results of this type are known for nonlinear equations and the authors know of no such results in case $r(t) \not 0$.

Our main result is contained in Theorem 2. In Theorem 3 we generalize the form of the above equation. We also include examples showing that some of our hypotheses are necessary.

2. Sufficient conditions for nonoscillation. Consider the equations

$$
\left(a(t) x^{\prime}\right)^{\prime}+q(t) x=0
$$

and

$$
\left(a(t) x^{\prime}\right)^{\prime}+q(t) f(x)=r(t)
$$

where $a, q, r:\left[t_{0}, \infty\right) \rightarrow R$ and $f: R \rightarrow R$ are continuous and $a(t)>0$. For reference, we now state Komkov's result [1, Theorem 2].

Theorem. Suppose there exists a $C^{1}$ function $u(t)$ defined on $\left[t_{1}, t_{2}\right]$ and a function $G(u)$ such that $G(u(t))$ is not constant on $\left[t_{1}, t_{2}\right], G\left(u\left(t_{1}\right)\right)=$ $G\left(u\left(t_{2}\right)\right)=0, g(u)=G^{\prime}(u)$ is continuous,

Received by the editors January 25, 1974 and, in revised form, March 27, 1974. AMS (MOS) subject classifications (1970). Primary 34C10; Secondary 34C15.

Key words and phrases. Nonoscillation.

${ }^{1}$ Supported by Mississippi State University Biological and Physical Sciences Research Institute. 


$$
\int_{t_{1}}^{t}\left\{a(t)\left[u^{\prime}(t)\right]^{2}-q(t) G(u(t))\right\} d t<0,
$$

and $g^{2}(u(t)) \leq 4 G(u(t))$ for $t$ in $\left[t_{1}, t_{2}\right]$. Then every solution of (1) must vanish on $\left[t_{1}, t_{2}\right]$.

We classify solutions of (2) in the following way. A solution $x(t)$ of (2) will be called nonoscillatory if there exists $t_{1} \geq t_{0}$ such that $x(t) \neq 0$ for $t \geq t_{1}$; the solution will be called oscillatory if for any given $t_{1} \geq t_{0}$ there exist $t_{2}$ and $t_{3}$ satisfying $t_{1}<t_{2}<t_{3}, x\left(t_{2}\right)>0$ and $x\left(t_{3}\right)<0$; and it will be called a $Z$-type solution if it has arbitrarily large zeros but is ultimately nonnegative or nonpositive.

Theorem 1. Suppose that

(i) equation (1) is nonoscillatory,

(ii) $r(t) \geq 0$ and $r(t) \not 0$ on any interval,

(iii) $x f(x) \geq 0$ for all $x$ and $f(x) \not 0$ on any interval, and

(iv) $[d(x f(x)) / d x]^{2} \leq 4 x f(x)$ for all $x \geq 0$.

Then no solution of (2) is oscillatory or nonnegative Z-type.

Proof. Suppose that $u(t)$ is an oscillatory or nonnegative Z-type solution of (2) and let $t_{1}$ and $t_{2}$ be consecutive zeros of $u(t)$ such that $t_{1}<t_{2}$ and $u(t)>0$ for $t$ in $\left(t_{1}, t_{2}\right)$. Since $u(t)$ is a solution of (2), we have

$$
\left(a(t) u^{\prime}(t)\right)^{\prime} u(t)+q(t) f(u(t)) u(t)=r(t) u(t)
$$

or,

$$
\left(a(t) u^{\prime}(t) u(t)\right)^{\prime}-r(t) u(t)=a(t)\left[u^{\prime}(t)\right]^{2}-q(t) f(u(t)) u(t) .
$$

Hence

$$
\int_{t_{1}}^{t_{2}}\left\{a(t)\left[u^{\prime}(t)\right]^{2}-q(t) f(u(t)) u(t)\right\} d t<0 .
$$

Letting $G(u)=f(u) u$, we see that the hypotheses of Komkov's theorem are satisfied so equation (1) is oscillatory. This contradiction completes the proof.

Example 1. The equation

$$
x^{\prime \prime}-9 f(x)=3(\sin t-1)\left(-3 \sin ^{2} t+\sin t-1\right), \quad t \geq 0
$$

where 


$$
f(x)= \begin{cases}x, & \text { if } x \geq 0 \\ x^{1 / 3}, & \text { if } x<0\end{cases}
$$

satisfies the hypotheses of our theorem but does possess the nonpositive Z-type solution $x(t)=(\sin t-1)^{3}$.

By putting a condition on the sign of $q(t)$ we can eliminate any nonpositive Z-type solutions and thus obtain the following nonoscillation result.

Theorem 2. If, in addition to the bypotheses of Theorem 1, $q(t) \geq 0$ for $t \geq t_{0}$, then all solutions of (2) are nonoscillatory.

Proof. It will suffice to show that (2) cannot have nonpositive Z-type solutions. Suppose that $x(t)$ is such a solution. Then

$$
\left(a(t) x^{\prime}(t)\right)^{\prime}=r(t)-q(t) f(x(t)) \geq 0 .
$$

Letting $t_{1}$ be a zero of $x^{\prime}(t)$ and integrating, we have $a(t) x^{\prime}(t) \geq 0$ for $t \geq t_{1}$, so $x^{\prime}(t) \geq 0$ for $t \geq t_{1}$ which is impossible for Z-type solutions.

The next example shows that $r(t)$ cannot be allowed to change signs.

Example 2. The equation

$$
x^{\prime \prime}+x / t^{3}=[t \cos (\ln t)+3 t \sin (\ln t)+\cos (\ln t)] / t^{4}, \quad t \geq 1,
$$

satisfies all the conditions of both of our the orems except that $r(t)$ changes signs. This equation has the oscillatory solution $x(t)=[\cos (\ln t)] / t$.

Remark. Suppose that $r(t) \leq 0$ and (iv) holds for $x \leq 0$. Then Theorem 1 would guarantee that no solution of (2) is oscillatory or nonpositive $Z$-type. The statement of Theorem 2 would remain the same but its proof would consist of ruling out the nonnegative $Z$-type solutions.

The proof of the following theorem is similar to the proofs above and will be omitted.

Theorem 3. Suppose $q(t) \geq 0$, (ii)-(iv) bold, $b: R \rightarrow R$ is continuous, and either

(v) $b(s) \leq 1$ and (i) bolds, or

(vi) for some constant $d>0, a(t) \geq d \geq b(s)$ and $x^{\prime \prime}+q(t) x=0$ is nonoscillatory.

Then no solution of

$$
\left(a(t) x^{\prime}\right)^{\prime}+q(t) f(x) b\left(x^{\prime}\right)=r(t)
$$


is oscillatory or nonnegative Z-type. If, in addition, $b(s) \geq 0$ for all $s$, then all solutions of (3) are nonoscillatory.

The remark following Example 2 also applies to Theorem 3.

\section{REFERENCES}

1. V. Komkov, A generalization of Leighton's variational theorem, Applicable Anal. 1 (1972), 377-383.

2. W. Leighton, Comparison theorems for linear differential equations of second order, Proc. Amer. Math. Soc. 13 (1962), 603-610. MR 25 \#4173.

DEPARTMENT OF MATHEMATICS, MISSISSIPPI STATE UNIVERSITY, MISSISSIPPI STATE, MISSISSIPPI 39762 